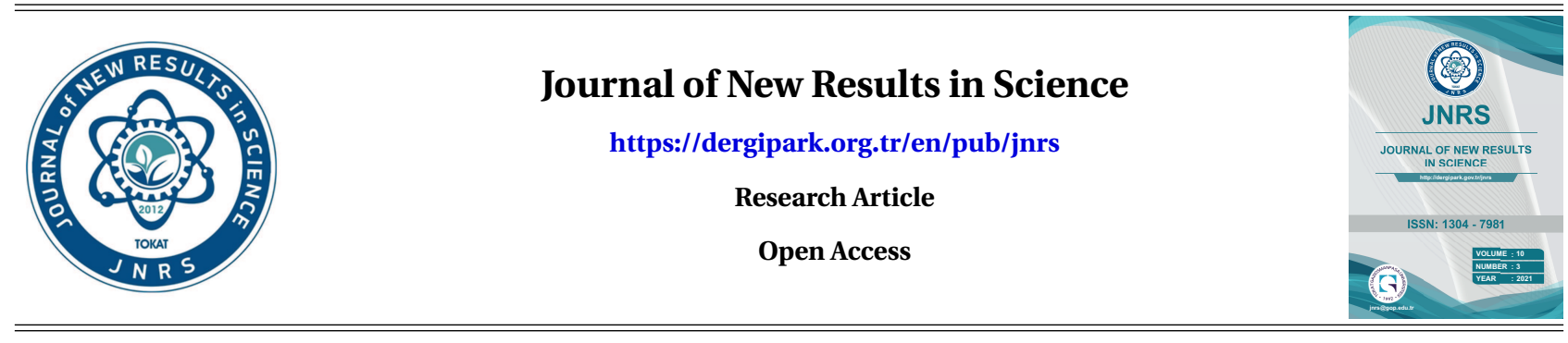

\title{
Conditional complements of sets and their application to group theory
}

\author{
Naim Çă̆man ${ }^{1}$ (D)
}

Keywords
Complement of a set,
Conditional
complements,
Inclusive complement,
Exclusive complement,
Complement subgroup

Subject Classification (2020): 03E75, $20 \mathrm{D} 05$.

\section{Introduction}

The concept of sets, whose borning dates back to late 1873, was proposed in 1874 by Georg Cantor (18451918). Sets are one of the most basic concepts in mathematics. They are now used in all areas of mathematics and form the basis of almost all mathematics. Since set-theoretical language is a very useful tool for describing and structuring mathematical objects, almost all mathematical concepts can be expressed via sets. This is considered one of the greatest achievements in mathematics. This achievement manifests that sets, which have practical mathematical tools, provide a foundation for mathematics.

A universal set, generally represented by $U$, is a fundamental set that contains all the elements of the considered sets. The complement of a set, which is an essential concept in set theory, consists of the elements of the universal set except for those of the set. In other words, for any subset $A$ of $U$, the complement of $A$, denoted by $A^{\prime}$, is defined as the set of all elements in the universe $U$ that are not in $A$. It means that

$$
A^{\prime}=U \backslash A
$$

In this work, we define two conditional complements of a set as new concepts of set theory, i.e., inclusive complement and exclusive complement. We then investigate the relationships between the two. We finally define complement subgroup of a group and give two questions about it.

\footnotetext{
${ }^{1}$ naim.cagman@gop.edu.tr (Corresponding Author)

${ }^{1}$ Department of Mathematics, Faculty of Arts and Sciences, Tokat Gaziosmanpasa University, Tokat, Turkey

Article History: Received: 02 Oct 2021 - Accepted: 10 Dec 2021 - Published: 31 Dec 2021
} 
For the basic definitions, theorems and properties of set theory, it can be recommend to look at the references [1,2].

Throughout this work, $U$ refers to a universe, $A^{\prime}$ is the complement of $A$ over $U$, and $A^{\prime \prime}$ denotes the complement of $A^{\prime}$.

\section{Inclusive Complement of Sets}

This section defines inclusive complement of a set and investigates its properties.

Definition 2.1. Let $A$ and $B$ be two subsets of $U$. Then, $B$-inclusive complement of $A$ is defined by

$$
A^{+B}:=A^{\prime} \cup B
$$

If $B=\{x\}$, then we will use $A^{+x}$ instead of $A^{+\{x\}}$. Therefore, " $x$-inclusive complement" is used for " $\{x\}$ inclusive complement".

Example 2.2. Let $U=\{0,1,2, \ldots, 10\}, A=\{0,2,4, \ldots, 10\}$, and $B=\{2,3,5,7\}$. Then,

$$
A^{+B}=\{1,2,3,5,7,9\}
$$

Example 2.3. Let us give some examples to show that the inclusive complement is used in daily life. Let $A=\{x \in U: p(x)\}$ and $B=\{x \in U: q(x)\}$ be two subsets of $U$. Then, $B$-inclusive complement of $A$ can be defined by

$$
A^{+B}=\{x \in U: \neg p(x) \vee q(x)\}
$$

where $\neg p$ is the negation of $p$. Thus,

a) Boss of a business: Call all uncalled customers, including my father.

b) Teacher in a classroom: Except for those who cannot take the exam for this course, those who are not successful in this course will not be able to participate in the weekend trip.

c) A note that is on the door of a shopping center: Those who have a positive Covid-19 test or those who do not have the Covid-19 vaccine cannot enter this building.

Proposition 2.4. Let $A$ and $B$ be any two subsets of $U$. Then,
i. $A^{\prime+B}=B^{\prime+A}$
ii. $A^{+B^{\prime}}=B^{+A^{\prime}}$

\section{Proof.}

Let us prove (i). The proof of (ii) is done in a similar way.

$$
\begin{aligned}
A^{\prime+B} & =A^{\prime \prime} \cup B \\
& =A \cup B \\
& =B^{\prime \prime} \cup A \\
& =B^{\prime+A}
\end{aligned}
$$


Proposition 2.5. Let $A$ be a subset of $U$. Then,
i. $A^{+\varnothing}=A^{\prime}$
iii. $U^{+A}=A$
v. $A^{+A^{\prime}}=A^{\prime}$
vii. $A^{+A}=U$
ii. $\varnothing^{+A}=U$
iv. $A^{+U}=U$
vi. $A^{\prime+A}=A$

Proposition 2.6. Let $A$ be a subset of $U$. Then,
i. $x \notin A \Rightarrow A^{+x}=A^{\prime}$
ii. $x \in A \Rightarrow\{x\}^{+A}=U$
iii. $x \notin A \Rightarrow\{x\}^{+A}=\{x\}^{\prime}$

Proposition 2.7. Let $A, B$ and $C$ be three subsets of $U$. Then, De Morgan's Laws are valid,
i. $(A \cup B)^{+C}=A^{+C} \cap B^{+C}$
ii. $(A \cap B)^{+C}=A^{+C} \cup B^{+C}$

\section{Proof.}

Let us prove (i). The proof of (ii) is done in a similar way.

$$
\begin{aligned}
(A \cup B)^{+C} & =(A \cup B)^{\prime} \cup C \\
& =\left(A^{\prime} \cap B^{\prime}\right) \cup C \\
& =\left(A^{\prime} \cup C\right) \cap\left(B^{\prime} \cup C\right) \\
& =A^{+C} \cap B^{+C}
\end{aligned}
$$

Proposition 2.8. Let $A, B$ and $C$ be any three subsets of $U$. Then,
i. $A^{+(B \cup C)}=A^{+B} \cup A^{+C}$
ii. $A^{+(B \cap C)}=A^{+B} \cap A^{+C}$

\section{Proof.}

Let us prove (ii). The proof of (i) can be done in a similar way.

$$
\begin{aligned}
A^{+(B \cap C)} & =A^{\prime} \cup(B \cap C) \\
& =\left(A^{\prime} \cup B\right) \cap\left(A^{\prime} \cup C\right) \\
& =A^{+B} \cap A^{+C}
\end{aligned}
$$

Proposition 2.9. Let $A$ and $B$ be two subsets of $U$. Then,
i. $\left(A^{+B}\right)^{+C}=A^{\prime+C} \cap B^{+C}$
ii. $\left(A^{+C}\right)^{+B}=A^{+B} \cap C^{+B}$

\section{Proof.}

Let us prove (i). The proof of (ii) can be done in a similar way.

$$
\begin{aligned}
\left(A^{+B}\right)^{+C} & =\left(A^{+B}\right)^{\prime} \cup C \\
& =\left(A^{\prime} \cup B\right)^{\prime} \cup C \\
& =\left(A^{\prime \prime} \cap B^{\prime}\right) \cup C \\
& =\left(A^{\prime \prime} \cup C\right) \cap\left(B^{\prime} \cup C\right) \\
& =A^{\prime+C} \cap B^{+C}
\end{aligned}
$$

Corollary 2.10. Let $A$ and $B$ be any two subsets of $U$. Then, $\left(A^{+B}\right)^{+B}=A^{++B}$. 
Remark 2.11. Let $A, B$ and $C$ be three subsets of $U$. Then, $\left(A^{+B}\right)^{+C} \neq\left(A^{+C}\right)^{+B}$.

Proposition 2.12. Let $A$ and $B$ be two subsets of $U$. Then,
i. $A^{+\left(B^{+C}\right)}=A^{+C} \cup B^{+C}$
ii. $A^{+\left(C^{+B}\right)}=A^{+B} \cup C^{+B}$

\section{Proof.}

Let us prove (i). The proof of (ii) can be done in a similar way.

$$
\begin{aligned}
A^{+\left(B^{+C}\right)} & =A^{\prime} \cup B^{+C} \\
& =A^{\prime} \cup\left(B^{\prime} \cup C\right) \\
& =\left(A^{\prime} \cup C\right) \cup\left(B^{\prime} \cup C\right) \\
& =A^{+C} \cup B^{+C}
\end{aligned}
$$

Remark 2.13. Let $A, B$ and $C$ be any three subsets of $U$. Then, $A^{+\left(B^{+C}\right)} \neq A^{+\left(C^{+B}\right)}$.

\section{Exclusive Complement of Sets}

This section defines exclusive complement of a set and investigates its properties.

Definition 3.1. Let $A$ and $B$ be two subset of $U$. Then, $B$-exclusive complement of $A$ is defined by

$$
A^{-B}:=A^{\prime} \backslash B
$$

If $B=\{x\}$, then we will use $A^{-x}$ instead of $A^{-\{x\}}$. Therefore, " $x$-exclusive complement" is used for " $\{x\}$ exclusive complement".

Example 3.2. Let $U=\{0,1,2, \ldots, 20\}, A=\{0,2,4, \ldots, 20\}$, and $B=\{2,3,5,7,11,13,17,19\}$. Then,

$$
A^{-B}=\{1,9,15\}
$$

Example 3.3. Let us give some examples to show that the exclusive complement is used in daily life. Let $A=\{x \in U: p(x)\}$ and $B=\{x \in U: q(x)\}$ be two subsets of $U$. Since $A^{\prime} \backslash B=A^{\prime} \cap B^{\prime}=(A \cup B)^{\prime}, B$-exclusive complement of $A$ can be defined by

$$
\begin{aligned}
A^{-B} & =\{x \in U: \neg p(x) \wedge \neg q(x)\} \\
& =\{x \in U: \neg(p(x) \vee q(x))\}
\end{aligned}
$$

where $\neg p$ is the negation of $p$. Thus,

a) Boss of a business: Call all uncalled customers, except my father.

b) Teacher in a classroom: Only those who could not take the exam for this course and those who took the exam for this course but did not fail the exam will participate in the weekend trip.

c) A note that is on the door of a shopping center: Only those who have a positive Covid-19 test or have not been vaccinated for Covid-19 cannot enter this building. 
Proposition 3.4. Let $A$ and $B$ be two subsets of $U$. Then, $A^{-B}=B^{-A}$.

Proof.

$$
\begin{aligned}
A^{-B} & =A^{\prime} \backslash B \\
& =A^{\prime} \cap B^{\prime} \\
& =B^{\prime} \cap A^{\prime} \\
& =B^{\prime} \backslash A \\
& =B^{-A}
\end{aligned}
$$

Corollary 3.5. Let $A$ be a subsets of $U$. Then, $A^{-x}=\{x\}^{-A}$ for all $x \in U$.

Proposition 3.6. Let $A$ be a subset of $U$. Then,
i. $A^{-\varnothing}=A^{\prime}$
iv. $A^{-A^{\prime}}=\varnothing$
ii. $A^{-U}=\varnothing$
v. $\varnothing^{-A}=A^{\prime}$
iii. $A^{-A}=A^{\prime}$
vi. $U^{-A}=\varnothing$

Proposition 3.7. Let $A, B$ and $C$ be three subsets of $U$. Then, De Morgan's Laws are valid,
i. $(A \cup B)^{-C}=A^{-C} \cap B^{-C}$
ii. $(A \cap B)^{-C}=A^{-C} \cup B^{-C}$

\section{Proof.}

Let us prove (i). The proof of (ii) is done in a similar way.

$$
\begin{aligned}
(A \cup B)^{-C} & =(A \cup B)^{\prime} \backslash C \\
& =\left(A^{\prime} \cap B^{\prime}\right) \cap C^{\prime} \\
& =\left(A^{\prime} \cap C^{\prime}\right) \cap\left(B^{\prime} \cap C^{\prime}\right) \\
& =\left(A^{\prime} \backslash C\right) \cap\left(B^{\prime} \backslash C\right) \\
& =A^{-C} \cap B^{-C}
\end{aligned}
$$

Proposition 3.8. Let $A, B$ and $C$ be three subsets of $U$. Then,
i. $A^{-(B \cup C)}=A^{-B} \cap A^{-C}$
ii. $A^{-(B \cap C)}=A^{-B} \cup A^{-C}$

\section{Proof.}

Let us prove (i). The proof of (ii) can be done in a similar way.

$$
\begin{aligned}
A^{-(B \cup C)} & =A^{\prime} \backslash(B \cup C) \\
& =A^{\prime} \cap(B \cup C)^{\prime} \\
& =A^{\prime} \cap\left(B^{\prime} \cap C^{\prime}\right) \\
& =\left(A^{\prime} \cap B^{\prime}\right) \cap\left(A^{\prime} \cap C^{\prime}\right) \\
& =\left(A^{\prime} \backslash B\right) \cap\left(A^{\prime} \backslash C\right) \\
& =A^{-B} \cap A^{-C}
\end{aligned}
$$


Proposition 3.9. Let $A$ and $B$ be two subsets of $U$. Then,
i. $\left(A^{-B}\right)^{-C}=A^{\prime-C} \cup B^{\prime-C}$
ii. $\left(A^{-C}\right)^{-B}=A^{-B} \cap C^{-B}$

\section{Proof.}

Let us prove (i). The proof of (ii) can be done in a similar way.

$$
\begin{aligned}
\left(A^{-B}\right)^{-C} & =\left(A^{+B}\right)^{\prime} \backslash C \\
& =\left(A^{\prime} \backslash B\right)^{\prime} \cap C^{\prime} \\
& =\left(A^{\prime} \cap B^{\prime}\right)^{\prime} \cap C^{\prime} \\
& =\left(A^{\prime \prime} \cup B^{\prime \prime}\right) \cap C^{\prime} \\
& =\left(A^{\prime \prime} \cap C^{\prime}\right) \cup\left(B^{\prime \prime} \cap C^{\prime}\right) \\
& =\left(A^{\prime \prime} \backslash C\right) \cup\left(B^{\prime \prime} \backslash C\right) \\
& =A^{\prime-C} \cup B^{\prime-C}
\end{aligned}
$$

Corollary 3.10. Let $A$ and $B$ be any two subsets of $U$. Then, $\left(A^{-B}\right)^{-B}=A^{\prime-B}$.

Remark 3.11. Let $A, B$ and $C$ be any three subsets of $U$. Then, $\left(A^{-B}\right)^{-C} \neq\left(A^{-C}\right)^{-B}$.

Proposition 3.12. Let $A$ and $B$ be two subsets of $U$. Then, $A^{-\left(B^{-C}\right)}=A^{-\left(C^{-B}\right)}$

\section{Comparison of Conditional Complements}

This section compares the inclusive complement and exclusive complement of a group and investigates the relationships between the two.

Proposition 4.1. Let $A$ and $B$ be any two subsets of $U$. Then,
i. $A^{+B}=\left(A^{\prime-B}\right)^{\prime}$
ii. $A^{-B}=\left(A^{\prime+B}\right)^{\prime}$

\section{Proof.}

Let us prove (i). The proof of (ii) can be done in a similar way.

$$
\begin{aligned}
\left(A^{\prime-B}\right)^{\prime} & =\left(A^{\prime \prime} \backslash B\right)^{\prime} \\
& =\left(A^{\prime \prime} \cap B^{\prime}\right)^{\prime} \\
& =A^{\prime} \cup B \\
& =A^{+B}
\end{aligned}
$$

Corollary 4.2. Let $A$ and $B$ be two subsets of $U$. Then,
i. $\left(A^{-B}\right)^{\prime}=A^{\prime+B}$
ii. $\left(A^{+B}\right)^{\prime}=A^{\prime-B}$

Proposition 4.3. Let $A$ be a subset of $U$. Then,
i. $A^{+\varnothing}=A^{-\varnothing}$
iii. $\left(\varnothing^{+A}\right)^{\prime}=U^{-A}$
ii. $\left(A^{+U}\right)^{\prime}=A^{-U}$
iv. $\left(A^{+A}\right)^{\prime}=A^{-A^{\prime}}$ 
Proposition 4.4. Let $A$ and $B$ be any two subsets of $U$. Then,
i. $\left(A^{+B}\right)^{-B}=A^{-B}$
ii. $\left(A^{-B}\right)^{+B}=A^{\prime+B}$

Proposition 4.5. Let $A$ and $B$ be any two subsets of $U$. Then,
i. $\left(A^{+B}\right)^{-C}=A^{-(B \cup C)}$
ii. $\left(A^{-B}\right)^{+C}=A^{\prime+(B \cup C)}$

Proposition 4.6. Let $A$ and $B$ be any two subsets of $U$. Then,
i. $\left(A^{-B}\right)^{+C}=\left(A^{-C}\right)^{+B}$
ii. $\left(A^{+B}\right)^{-C}=\left(A^{+C}\right)^{-B}$

Proposition 4.7. Let $A$ and $B$ be any two subsets of $U$. Then,
i. $A^{+B} \cup A^{-B}=A^{+B}$
ii. $A^{+B} \cap A^{-B}=A^{-B}$

Corollary 4.8. Let $A$ and $B$ be any two subsets of $U$. Then, $A^{-B} \subseteq A^{+B}$.

\section{Complement Subgroup of a Group}

This section defines complement subgroup of a group. For the basic definitions, theorems and properties of group theory, please look at the references $[3,4]$.

Definition 5.1. Let $G$ be a group, $H$ and $K$ be two subgroups of $G$. If $H^{+K}$ is a subgroup of $G$, then $H^{+K}$ is called complement subgroup of $G$. Here, $H^{+K}:=(G \backslash H) \cup K$.

Proposition 5.2. Let $G$ be a group with identity $e$. Then,

i. $G^{+e}$ is a complement subgroup of $G$.

ii. $\{e\}^{+e}$ is a complement subgroup of $G$.

\section{Proof.}

Let $G$ be a group with identity $e$. Then,

i. $G^{+e}=G^{\prime} \cup\{e\}=\varnothing \cup\{e\}=\{e\}$.

ii. $\{e\}^{+e}=\{e\}^{\prime} \cup\{e\}=G$.

Proposition 5.3. Let $G$ be a group and $H$ be a subgroup of $G$. Then, $H^{+H}$ is a complement subgroup of $G$.

\section{Proof.}

$H^{+H}=H^{\prime} \cup H=G$.

Proposition 5.4. Let $G$ be a group, $H$ and $K$ be two subgroups of $G$. If $H$ is a subgroup of $K$, then $H^{+K}$ is a complement subgroup of $G$.

Proof.

$H^{+K}=H^{\prime} \cup K=G$.

Example 5.5. $2 \mathbb{Z}$ and $4 \mathbb{Z}$ are two subgroups of $4 \mathbb{Z}$. Since $4 \mathbb{Z}$ is a subgroups of $2 \mathbb{Z}$, then $4 \mathbb{Z}^{+2 \mathbb{Z}}$ is a complement subgroup of $\mathbb{Z}$. 


\section{Questions}

Questions 1. Let $\mathrm{G}$ be a group whose identity is e. Assume that $H$ is a nontrival subgroups of $G$ that is $H \neq G$ and $H \neq\{e\}$. Under these conditions, is there any group $\mathrm{G}$ such that $H^{+e}$ is a complement subgroup of $\mathrm{G}$ ?

Let's generalize this question a little more.

Questions 2. Assume that $H$ and $K$ are two subgroups of a grup $G$ such that $K \nsubseteq H$ and $H \nsubseteq K$. Under these conditions, is there any group G such that $K^{+H}$ is a complement subgroup of G?

\section{Conclusion}

In this work, we first defined two conditional complements of a set, namely inclusive complement and exclusive complement. By comparing these the two, we then explored the relationships between them. We also applied these complements to the group theory and defined complement subgroup of a group. Finally, we finished the study with two interesting questions about the subject.

\section{Author Contributions}

The author read and approved the last version of the manuscript.

\section{Conflicts of Interest}

The author declares no conflict of interest.

\section{References}

[1] P. R. Halmos, Naive set theory, D. Van Nostrand Company, Inc., New York, 2011.

[2] J. Ferreirós, Labyrinth of thought: A history of set theory and its role in modern mathematics, Birkhäuser, Basel, 2007.

[3] D. S. Dummit, R. M. Foote, Abstract algebra, Wiley, Hoboken, NJ, 2004.

[4] T. Hungerford, Algebra, Springer, New York, 2003. 